\title{
Accuracy of a Self-Collection Kit for the Microbiological Study of the Vaginal Content
}

\author{
Mauro Romero L. Passos ${ }^{1}$, Renata Q. Varella $^{1}$, Nero A. Barreto ${ }^{1}$, Maria Luiza Garcia ${ }^{2}$ and Paulo C. Giraldo ${ }^{3}$ \\ ${ }^{1}$ STD Sector at Fluminense Federal University; ${ }^{2}$ Epidemiology Department at Fluminense Federal University; Niterói, RJ; ${ }^{3}$ Gynecology and \\ Obstetrics Depatment at University of Campinas; Campinas, SP, Brazil
}

\begin{abstract}
Diagnosis of vaginal discharge is frequently performed in an empirical way, leading to inadequate treatment. This study tested the accuracy of a self-collection kit for microbiological study of the vaginal content. One hundred and forty-two women of Family Health Program units in Niterói and Piraí cities were enrolled in order to have their vaginal content studied. A brief explanation and a self-collection kit were provided in order to sample the vaginal content. The self-collection kit was composed of one empty plastic tube, two glass slides, a long handle cytobrush, an identification card and guideline notes. The vaginal sample was applied on the glass slides by the women and stained by Gram technique. A second sampling was done by the medical personnel. The microbiological diagnosis in a blinded analysis was made under optical microscopy. A validation diagnosis test was done taking the medical collection results as a gold standard. A total of 106 women had followed the protocol and were included in the study. Microbiological analysis was unsatisfactory in 12 cases ( 6 cases of self-collection material and 6 cases of medical collection). The microbiological analyses in the self-collection and in the medical collection material were respectively: bacterial vaginosis in $21.7 \%$ and $\mathbf{1 7 . 9 \%}$, non bacillar flora in $\mathbf{1 0 . 3 \%}$ and $11.3 \%$, vaginal trichomoniasis in $\mathbf{5 . 6 6 \%}$ and $5.6 \%$, candidiasis in $3.78 \%$ and $2.8 \%$ and a normal microbiota in $52.8 \%$ and $56.6 \%$. The Kappa coefficient suggested a "very good correlation" of the microbiological results between the two methods of collection $(\mathrm{K}=0.7945)$. The selfcollection kit provides samples for microbiological analysis of the vaginal microbiota as good as medical collection.
\end{abstract}

Key-Words: Self-collection kit, vaginitis, diagnosis, vaginal discharge, bacterial vaginosis, vaginal flora.

Vulvovaginitis (VV) is considered the most common problem in Gynecology, comprising more than 10 million visits in the United States annually. It occurs when the normal vaginal ecosystem is broken and can be present in women worldwide [1].

The physiological vaginal content is constituted by diverse compounds such as water, epithelial cells, proteins, carbohydrates and microorganisms. The greater part of the vaginal secretion is originated from transudation through the capillaries of the vaginal walls [1]. The physiological vaginal microbiota (flora) is composed mainly by Lactobacillus acidophilus [2], which helps the vaginal ecosystem balance. Bacterial vaginosis (BV), vaginal candidiasis and trichomoniasis represent the most common vaginal infections [3-6], however, there are many cases of vaginal abnormality without a precise diagnosis.

The lack of Lactobacillus sp. in the vaginal content is a crucial factor, leading women to different infections. The easiest and less expensive way to check the vaginal flora is the microbiological analysis using optical microscopy where the microorganisms were stained by Gram technique. It provides to the microbiologist a good tool to analyze the morphology of the microorganisms responsible for vaginitis

Received on 15 September 2006; revised 22 March 2007.

Address for correspondence: Dr. Mauro Romero Leal Passos. Setor de DST. Departamento de Microbiologia e Parasitologia. Universidade Federal Fluminense. Rua Professor Hernani Melo, 101, anexo. São Domingos, Niterói - RJ. Zip code: 24210-130. Phone: 21 2629-2507 - 21 9888-2897. Fax: 21 2629-2506. E-mail: maurodst@vm.uff.br.

The Brazilian Journal of Infectious Diseases

2007;11(2):249-253. (C) 2007 by The Brazilian Journal of Infectious Diseases and Contexto Publishing. All rights reserved.
[7] or the abnormal ecosystem. In the usual clinical activity, the gynecologist or the general practitioner (GP) may find difficult to establish the etiologic diagnosis for the vaginal discharge. Usually, the diagnoses of the vaginal discharge are done using the clinical observation or anamnesis only. Keywords such as itching, burning and bad odor are used for diagnosis, leading to inadequate treatment [8] if it is not followed by microbiological analysis. Lots of women modify the vaginal content douching or having sexual intercourse before the gynecological exam, which makes it more difficult to be analyzed. The gynecologist can misdiagnose the vaginal discharge if take in consideration the anamnesis and gynecological exam only. Microbiological analysis using Gram technique is easy, inexpensive and very important for a correct and early diagnosis of the vaginal discharge. The use of an inadequate medication can cause unnecessary costs, side effects or worse diseases. Vaginal inflammation and/or infection induce migration of HIV infected or susceptible cells into the lesion site. The migration would increase infectivity or susceptibility to HIV. Cervicitis and vulvovaginitis are, certainly, important contributors in this scenario [9].

Although the most frequent causes of $\mathrm{VV}$ are represented by $\mathrm{BV}$, fungi infections and trichomoniasis, it is worth remembering that in approximately $10 \%$ of cases, another etiology is present. In those cases, vaginal microscopy using Gram stain is still the most practical diagnostic method [10].

It is necessary to look for a simple modus operandis, with widespread use, acceptable efficacy and economically accessible to be used in the diagnosis of vaginitis.

The self-collection of biological material, preformed by the patients themselves, is not a new issue. Urine, feces, and sperm are routinely collected by the patients. It is practical 
and allows agility in clinical investigation. However, instructions are needed to perform an appropriated selfcollection of the material. The effectiveness of the selfcollection technique is already proven in the field of molecular biology, but not for microbiological studies of the vaginal discharges.

The direct analysis by the staining of the self-collected material with the lamina prepared also by the patient is still something innovative. The objectives of this study were to test the effectiveness of a kit for self-collection of vaginal content for the study of the vaginal microbiota using Gram stained microscopy, comparing to the material collected by medical personnel.

\section{Materials and Methods}

The instructions and the self-collection kits were offered to the participants of the study by the general practitioner of the Family Health Program in the cities of Niterói and Piraí, Rio de Janeiro State, Brazil. The self-collection kit was composed of: instructions notes, identification card, long brush $(18 \mathrm{~cm})$, hollow plastic tube $(1 \mathrm{x} 11 \mathrm{~cm})$ and a slide of glass identified.

All the participants signed the consent form and were informed to perform the collection in domiciliary environment and to return to the health care unit in order to have their vaginal content sampled by medical personnel. The medical personnel used a similar kit for the collection of vaginal material, except for the vaginal speculum in the place of the hollow plastic tube. Validation test study for diagnosis was performed using the medical personnel collection as gold standard.

Target population: Adult women attended in the primary health care units of the Unified Health System in the cities of Niterói and Piraí, Rio de Janeiro State, Brazil, complaining or not of vaginal discharge. The exams were masked from the microbiologist who performed the microscopic diagnosis in the laboratory of sexually transmitted diseases, Department of Microbiology and Parasitology at the Fluminense Federal University. The study was carried out from March to September 2002 with sexually active women. Vaginal bleeding, usage of antibiotics or any vaginal product 30 days before the beginning of the study, and sexual intercourse during the last 2 days were used as exclusion criteria. The frequency of etiological diagnosis of vulvovaginitis was established in a blind study and the results for each woman were determined in the two groups according to the method of collection. We estimated the sensitivity, the specificity, and the predictive values (positive and negative) for self-collected results taking the medical collected results as a "gold standard".

Kappa coefficient evaluated the agreement between the two methods.

\section{Vaginal Microbiological Diagnosis}

Normal (LB predominance, no inflammation, few lysed cells), abnormal (lack of LB, no or discrete inflammation, no specific pathogenic agent), vaginal candidiasis (presence of hyphas and inflammation), trichomoniasis (presence of Trichomonas vaginalis and severe inflammation), and bacterial vaginosis (Nugent's criteria) [7]. The study was approved by the Ethics Research Committee of the Medical Sciences Center of the Hospital Universitário Antônio Pedro, Fluminense Federal University.

\section{Results}

A total of 142 kits for self-collection were distributed (80 in Niterói and 62 in Piraí). One hundred and six women (74.65\%) completed the study protocol including 58 women from Niterói (54.72\%) and 48 from Piraí (48.28\%). The mean age of the participants was 30.73 years. Ten women (9.43\%) reported that were not having sexual intercourse during the study and 17 (16.03\%) did not use any anti-conception method even while having sexual intercourse routinely. Twenty women (18.87\%) reported never having a gynecologic exam for Pap smear collection before. The vaginal material collected by the medical personnel identified 60 (56.6\%) cases of normal flora (NF), 12 (11.32\%) cases of abnormal flora (AF); 19 (17.93\%) cases of VB; 6 (5.66\%) cases of trichomoniasis, 3 (2.83\%) cases of candidiasis and 6 (5.66\%) cases of unsatisfactory material for an adequate microbiological analysis (Table 1). The self-collected material microbiological analyses have shown $52.8 \%$ of NF, $10.38 \%$ of AF, $21.7 \%$ of VB, $5.66 \%$ of TV, $3.78 \%$ of $C V$ and also 6 (5.66\%) cases of inappropriate material for microscopy analysis.

The Kappa coefficient suggested a "very good correlation" of the microbiological results between the two methods of collection ( $\mathrm{K}=0.7945)$, using the results of the medical personnel collection as a gold-standard (Table 2).

The sensitivity, specificity, positive predictive value (PPV) and negative predictive value (NPV) are showed at Table 3.

\section{Discussion}

This is probably the first study comparing vaginal flora using a self collection kit. This kit could give more options for patients to have their vaginal content analyzed without going to the gynecologist. Urine, feces, sperm are usually collected by the patients, however, the vaginal content is not. In a similar study started in 1997 using the same kit, we compared the self-collection method of vaginal content by patients of high socioeconomic status only [11].

The main objective of the study was the development of a kit that would work safely, providing user satisfaction and adequate material collection in population groups with low socioeconomic status and difficult access to health care centers. Because of that, the authors evolved to the Pilot-2 study. This study included women attended by the Family Health Program in the city of Niterói, Rio de Janeiro State [12]. In this study, 23 women completed the study protocol, but in 3 cases of self-collection, the material was insufficient for the analysis. Of these 23 women, 2 (8.7\%) also had insufficient material for the cytologic analysis when collected by the medical personnel. The results were similar in 19 of the 23 women (83.6\%). 
Table 1. Vaginal microbiological diagnosis according to the method of collection

\begin{tabular}{ccc}
\hline Diagnosis & $\begin{array}{c}\text { Self-collection } \\
\mathbf{N}(\%)\end{array}$ & $\begin{array}{c}\text { Medical collection } \\
\mathbf{N}(\%)\end{array}$ \\
\hline NF & $56(52,83)$ & $60(56,60)$ \\
VB & $23(21,7)$ & $19(17,93)$ \\
AF & $11(10,38)$ & $12(11,32)$ \\
TV & $6(5,66)$ & $6(5,66)$ \\
CV & $4(3,78)$ & $3(2,83)$ \\
USM & $6(5,66)$ & $6(5,66)$ \\
Total & $106(100)$ & $106(100)$ \\
\hline
\end{tabular}

$\mathrm{CV}=$ candidiasis vaginal; USM=unsatisfactory material; $\mathrm{AF}=$ abnormal flora; $\mathrm{NF}=$ normal flora; $\mathrm{TV}=$ trichomoniasis vaginal; $\mathrm{BV}=$ bacterial vaginosis.
Table 2. Microbiological diagnosis in the vaginal content sampled by patients self-collection and medical collection

\begin{tabular}{lccccccc}
\hline $\begin{array}{l}\text { Self-collection } \\
\text { Frequence } \\
\text { of cases }\end{array}$ & CV & USM & AF & NF & TV & VB & T \\
\cline { 2 - 8 } & 3 & 0 & 1 & 0 & 0 & 0 & 4 \\
CV & 0 & 3 & 1 & 2 & 0 & 0 & 6 \\
USM & 0 & 1 & 7 & 2 & 1 & 0 & 11 \\
AF & 0 & 1 & 0 & 55 & 0 & 0 & 56 \\
NF & 0 & 0 & 0 & 1 & 5 & 0 & 6 \\
Trichomoniasis & 0 & 1 & 3 & 0 & 0 & 19 & 23 \\
BV & 3 & 6 & 12 & 60 & 6 & 19 & 106 \\
Total & & &
\end{tabular}

$\mathrm{CV}=$ candidiasis vaginal; USM=unsatisfactory material; $\mathrm{AF}=$ abnormal flora; $\mathrm{NF}=$ normal flora; $\mathrm{TV}=$ trichomoniasis vaginal; $\mathrm{BV}=$ bacterial vaginosis. Kappa coefficient=0.7945. Confidence interval (95\%): 0.699-0.891.

Table 3. Sensitivity, specificity, PPV, NPV of vaginal microbiological diagnosis sampled by self-collection kit

\begin{tabular}{lcccc}
\hline Diagnosis & $\begin{array}{c}\text { Sensitivity } \\
\text { \% (IC 95\%) }\end{array}$ & $\begin{array}{c}\text { Specificity } \\
\text { \% (IC 95\%) }\end{array}$ & PPV (\%) & NPV (\%) \\
\hline NF & $91(84.7-98.7)$ & $97(93.6-100)$ & 98 & 90 \\
AF & $58(30.4-86.2)$ & $95(91.7-99.8)$ & 63 & 94 \\
VB & $100(30.4-86.2)$ & $95(91-99.8)$ & 82 & 100 \\
CV & $100(100-100)$ & $99(97-100)$ & 75 & 99 \\
TV & $83(53.5-100)$ & $99(97-100)$ & 83 & 99 \\
USM & $50(10-90)$ & $97(93.7-100)$ & 50 & 97 \\
\hline
\end{tabular}

Teste exato de Fisher; PPV=positive predictive value; NPV=negative predictive value; $\mathrm{CV}=$ candidiasis vaginal; $\mathrm{USM}=$ unsatisfactory material; $\mathrm{AF}=$ abnormal flora; $\mathrm{NF}=$ normal flora; $\mathrm{TV}=$ trichomoniasis vaginal; $\mathrm{BV}=$ bacterial vaginosis.

Figure 1. In this figure we observe the hollow plastic tube replacing the speculum. It provides an appropriate penetration of the long brush in the vagina. This method assures that the material can be collected from the vaginal fornix by the patients.
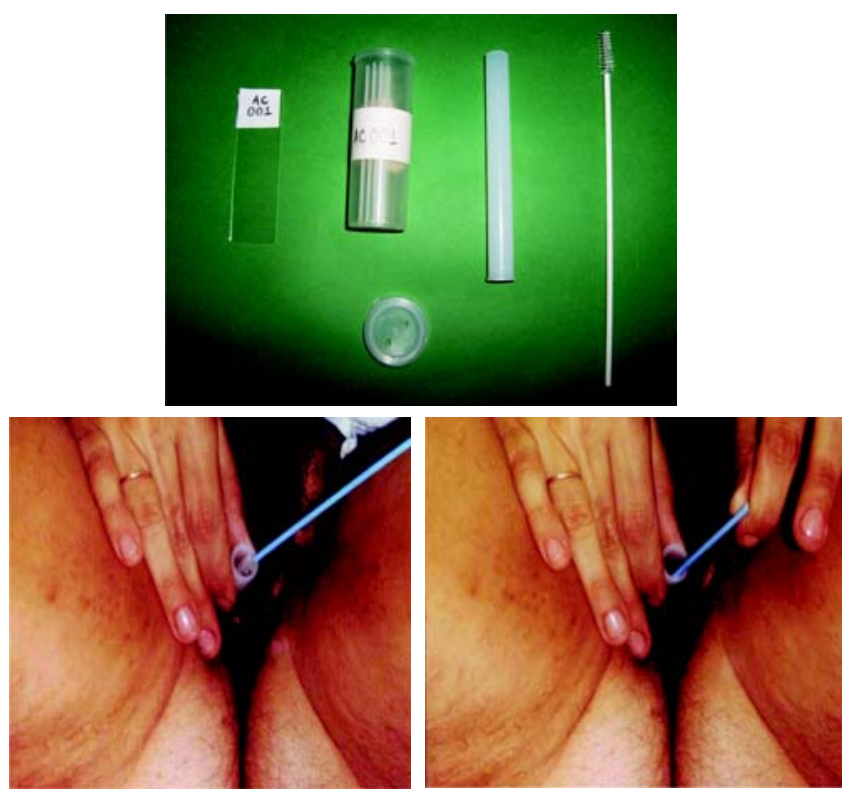

Bacterial vaginosis was detected in 5 women (20.83\%). The result that intrigued the authors was the finding that 3 women $(13 \%)$ were never submitted to a gynecologic examination. After that they performed the self-collection, these women were examined with no problems. They reported that they were afraid of the exam [12].

We found difficult to compare our findings because the method, to our knowledge, is unique and was idealized by ourselves. However, a common thought among researchers is that the method of self-collection can be of great help in the diagnosis of female genital infections.

However, the majority of the publications we found report self-collection for molecular biology purposes. These techniques are based in the detection and analysis of DNA and in our settings are difficult to perform and of high cost for the Brazilian population.

Verhoeven et al.[13] analyzed the method of self-collection of the content of the vaginal introitus to test the presence of Chlamydia trachomatis by the polymerase chain reaction (PCR) technique. The conclusion was that women are confidently able to collect material of herself and therefore to assist more responsibly in the preservation of her sexual health.

Knox et al.[14], working also with self-collected material and molecular biology methods for Neisseria gonorrhoeae, Chlamydia trachomatis and Trichomonas vaginalis in 
women of a central urban area of Australia, concluded that the self-collection of vaginal content for women living in a remote area is an acceptable and sensible alternative to test for microorganisms that cause sexually transmitted diseases (STD).

Holland-Hall et al.[15] studied adolescents in a correctional institution of Pennsylvania, in the United States of America, to evaluate the use and acceptance of self-collected vaginal swab in the molecular biology test for gonococcus, chlamydia, and trichomonas. They found exams positive for trichomonas in $11.7 \%$, for chlamydia in $11.3 \%$ and for gonococcus in $8.5 \%$. The method was accepted by $95 \%$ of the adolescents. The authors concluded that self-collection of vaginal content was highly acceptable among adolescents and that it may increase greatly the detection of those diseases when the pelvic examination is not performed.

Hsieh et al.[16] investigated the preference, with respect to comfort, of the self-collection of vaginal swabs and urine in military women. They concluded that self-collection of vaginal content with swab for Chlamydia trachomatis detection with molecular biology techniques can be a feasible alternative for the collection of urine in situations where stocking and transport are concerns. In the study, the main results were: $90.8 \%$ of women reported feeling comfortable in collecting the first urine and $69.9 \%$ reported feeling comfortable self-collecting vaginal content with swab.

However we found a study similar to ours, published in 2003 [17]. The study did not mention the timing and it studied pregnant women attended in the University Hospital of Pennsylvania, and resident women in the city of Philadelphia with no more than 12 weeks of lack of menstrual periods. The authors recruited women in one private and in one public health clinic. Self-collected specimens were compared to specimens collected by nurses trained for the study.

The two vaginal exams were performed introducing the swab approximately one inch inward the vaginal introitus. The materials collected were placed in appropriate recipients with no liquid environment. Both collections with swab were placed on glass lamina by the nurse trained for the study. The lamina were sent to the clinical microbiology laboratory of the study hospital to be colored by the Gram method and analyzed in a masked way by two microbiologists.

The study intended to compare the results of the selfcollected material versus the health personnel-collected, and the results of the microbiologists for the diagnosis of bacterial vaginosis in pregnant women.

The number of participants was 32 pregnant women.

In the comparison of the self versus health personnel collected material, there was 1 over 32 cases of discordance (3.12\%). Additionally, in the comparison of the results of the microbiologists, there was only one case of discordance.

Although this study was about the bacteriological evaluation of the vaginal content using the Gram stain method and it showed that women can collect her vaginal content, in our opinion, there is one inconvenient aspect to be considered. Women were required immediately after collecting to give the swab to one trained health personnel, in order to him prepare the lamina. This makes necessary that the patient collects the vaginal material inside a health care unit facility.

The original contribution of our study is the development of a kit that the woman herself collects and prepares the lamina to be stained in a domiciliary environment. We demonstrated the ability of the women attending a Unified Health Care System of two cities (Niterói e Piraí) in selfcollecting vaginal content and placing such material on a glass lamina to be colored a posteriori by the Gram stain.

When we have only the swab with the vaginal content, it has to be handled immediately for the preparation of the lamina, as in the case of Nelson et al study [17].

In the case of collection and lamina preparation made by the woman herself, the lamina can be analyzed days after, avoiding the immediate contact with the health care unit. It can even be sent through mail by people with difficult access to medical centers. In this way, when the patient goes to the health care unit to check the results of the exam, she will find important information about her vaginal microbiota.

The health care team, having no conditions to collect material for the study of vaginitis during the gynecologic examination, can make use of a basic element of a better quality, in the case of a self-collected material had being previously collected.

In the other side, women with vulvovaginitis symptoms can collect themselves vaginal materials and by the time of the medical visit, they would have the primary analysis of the vaginal discharge.

Some gynecologists, even in private practice, do not have the conditions necessary to perform a fresh exam or bacterioscopy during the medical visit. This is true for developing and developed countries.

It deserves notice the study of Gomes [18] that evaluated 124 women with complaints of vaginal discharge. The study intended to evaluate the sensitivity, specificity and the predictive values of the diagnosis of the vaginal discharge based on the clinical examination with vaginal speculum or based on complaints of the patient. The conclusion is that the clinical exam with the speculum and the symptoms, when taken alone, presented inadequate results in the correct diagnosis of vaginal discharge. With this data, once again, we have to remember the usefulness of a minimum laboratory workout in order to have a better diagnosis of the vaginal discharge complaints.

Another information that should be taken in consideration relates to the recruitment of women for the gynecologic exam. About $18 \%$ of women in our study were not submitted to a gynecologic examination until the present study. After the study where they collected their own vaginal content, without major problems and spontaneously, they started visiting the gynecologist. This result alone made us believe that our study was of great value. 
No women in the study presented a harmful effect on health caused by the material collection. Additionally, emotionally, we had no knowledge of any disturbance, although the study did not include any specific question about it.

The total number of unsatisfactory material for the microbiologic analysis was 12 (11.3\%). Of those, 6 were in the self-collected material and 6 in the health personnel collected.

Unsatisfactory material is considered when the stained cells on the slides provide scarce material, precluding an adequate analysis in the lamina of microbiological morphotypes and of epithelial cells. In the case of excess of material in the lamina, the analysis is also disturbed, due to impossibility to identify clearly the components of the vaginal content. These errors in the collection, although rare, may happen in any gynecologic unit.

Our study showed that more than $50 \%$ of the women presented normal vaginal content, in other words, with equilibrium of microbiota.

The self-collected and the health personnel collected material demonstrated statistically similar results for normal results and it is the same for abnormal results.

The majority of the women in the study was not looking for health care and was not with complaints of vaginal discharge. The finding of $20 \%$ of bacterial vaginosis and $5.7 \%$ of trichomoniasis deserves special attention of the public health authorities. These are pathologies that facilitate complications in the reproductive health of women. Trichomoniasis is a classic STD. Women with one STD have a higher risk of having another one or more.

The method presented in this study did not intend in any moment to exclude the medical examination, since we know that genital infection can co-exist with other pathologic processes, even more serious.

However, we propose that our method can benefit a great group of women that spent the whole life without gynecologic examination. Furthermore, when we diagnose vaginitis, we can create opportunities for other diagnosis of gynecologic diseases since women will be inside the basic health care units (at least to receive the results of the exam), units which are responsible for the global attention on the population health.

We find ideal to perform a large scale, multi-centric study with the present methodology. The results demonstrated that the kit of self-collection was effective, with satisfactory sensitivity and specificity, sufficient to consider this a good method with high correlation with the medical collected material for the diagnosis of vaginitis. The self-collection method is easy to perform, has low cost and was well accepted. This can represent an opportunity to recruit women who were never submitted to a gynecologic examination, especially, those women with limited access to the health care system.

\section{Acknowledgements}

Niterói and Piraí Family Health Programs.

\section{References}

1. Paavonen J. Physiology and ecology of the vagina. Scand J Infect Dis 1983;31:485-91.

2. Michel R.P., Bonsal R.W., Warner P. Human vaginal secretion: volatile fatty acid content. Science 1974;186:1217.

3. American College of Obstetrics and Gynecology. Vaginitis. Int J Gynecol Obstet 1996;53:271-80.

4. Escheanbach D.A. Vaginal infection. Clin Obstect Gynecol 1983;26:1-31.

5. Holmes K.K., Stranmm W.E. Lower genital tract infection in women. In: Holmes K.K., et al. Sexually Transmitted Diseases. New York: Mc Graw-Hill, 1999. p 761-81.

6. Fleury F.J. Adult vaginitis. Clin Obstct Gynecol 1981;24(2):40711.

7. Nugent R.P, Krohn M.A, Hillier S.L. Reliability of diagnosis of bacterial vaginosis is improved by a standardized method of Gram stain interpretation. J Clin Microbiol 1991;29:297-301.

8. Giraldo P.C., Ribeiro Filho A.D., Simões J.A., et al. Vulvovaginites - aspectos habitualmente não considerados; J Bras Ginec 1997;107(4):89-93.

9. Miranda S.D., Linhares I.M., Fonseca I.M., et al. Infecção pelo HIV e flora bacteriana vaginal. Rev Ginecol Obstet 1997;8:1036.

10. Landers D.V. Vaginitis/Cervicitis - Diagnosis and treatment options in a limited resource environment. Women's Health Issues 1996;6:342-8.

11. Passos M.R.L., Barreto N.A., Azevedo P.M.C., et al. Perfil da microbiota vaginal de mulheres de classe baixa de Niterói: comparativo de bacterioscopia pelo Gram X citologia corada e autocoleta X coleta por médico (estudo piloto 2). J bras Doenças Sex Transm 2000;12(5):103.

12. Passos M.R.L., Barreto N.A. Giraldo P.C., et al. Profile of vaginal flora in lower class women in Niterói: Comparative of bacterioscope by Gram X Pap smear and self-collected X medical collected (pilot survey 2). 6th Annual Meeting of International Infections Diseases. Society Obstetrics and Gynecology. USA, Chicago, April, 27-29; 2001. Program agenda, abstract p.18

13. Verhoeven V., Avonts D., Royen P.V., Denekens J. Self-collection of vaginal swab specimens: the patient's perception. STD 2002;29(7):426.

14. Knox J., Tabrizi S.N., Miller P., et al. Evaluation of self-colleted samples in contrast to practioner-collected samples for detection of Chlamydia trachomatis, Neisseria gonorrhoeae, and Trichomonas vaginalis by polymerase chain reaction among women livin in remote áreas. STD 2002;29(11):647-54.

15. Holland-Hall C.M., Wiesenfeld H.C., Murray P.J. Self-collected vaginal swabs for the detection of multiple sexually transmitted infections in adolescent girls. J Pediatr Adolesc Gynecol 2002;15:307-13.

16. Hsieh Y-H., Howell M.R., Gaydos J.C., et al. Preference among female army recruits for use of self-administrated vaginal swabs or urine to screen for Chlamydia trachomatis genital infections. STD 2003;30(10):769-73.

17. Nelson D.B., Bellamy S., Gray T.S., Nachamki I. Self-collected versus provider-collected vaginal swabs for the diagnosis of bacterial vaginosis: An assessement of vality and realibility. Journal of Clinical Epidemiology 2003;56:862-6.

18. Gomes F.A.M. Valor do Exame Clínico Especular e da Anamnese para o Diagnóstico do Corrimento Vaginal 2003. Tese (Doutorado). Faculdade de Ciências Médicas - UNICAMP. (Volume I). p. 31-2. 